\title{
A method for the inclusion of physical activity-related health benefits in cost-benefit analysis of built environment initiatives
}

Belen Zapata-Diomedia

Lucy, Gunn ${ }^{b}$

Billie, Giles-Corti ${ }^{b}$

Alan, Shiellc

J Lennert, Veerman ${ }^{a, d, ~ e ~}$

a The University of Queensland, School of Public Health, Herston Road, Herston, 4006, Brisbane, Queensland, Australia

${ }^{b}$ RMIT University, Healthy Liveable Cities Group, Centre for Urban Research, Melbourne, 3000, Victoria, Australia

c La Trobe University, Department of Public Health, Plenty Road, Bundoora, 3083, Victoria, Australia

${ }^{\mathrm{d}}$ Cancer Council NSW, Woolloomooloo, 2011, Sydney, NSW, Australia

e Sydney Medical School, Sydney, 2006, NSW, Australia

Corresponding author: Belen Zapata-Diomedi, b.zapatadiomedi@uq.edu.au 


\section{Abstract}

The built environment has a significant influence on population levels of physical activity (PA) and therefore health. However, PA-related health benefits are seldom considered in transport and urban planning (i.e. built environment) cost-benefit analysis. Cost-benefit analysis implies that the benefits of any initiative are valued in monetary terms to make them commensurable with costs. The aim of this study was to explore a method for the incorporation of monetised PA-related health benefits in cost-benefit analysis of built environment interventions. Firstly, we estimated the change in population level of PA attributable to a change in the built environment. Then, changes in PA were translated into monetary values, to obtain functions that directly link changes in features of the built environment to monetised health values. For the first step we used estimates from the literature. For the second step we used the proportional multi-state multi-cohort life table model to predict changes in healthadjusted life years and health care costs as a function of changes in PA. Finally, we monetised health-adjusted life years using the value of statistical life year. These methods could be adapted to assess the health and economic impacts of specific urban development scenarios by working in collaboration with urban planners.

Key words: cost benefit analysis; physical activity; health; built environment; urban planning; transport; active transport 


\section{List of abbreviations}

BE

CBA

PA

SP

VSL

VSLY

WTP
Built environment

Cost-benefit analysis

Physical activity

Stated preferences

Value of statistical life

Value of statistical life year

Willingness to pay 


\section{Introduction}

It is now well established that city and transport planning influence the health and wellbeing of urban populations (1). Worldwide, transport and land-use policies have contributed to the increasing burden of non-communicable diseases and injuries, mainly via physical inactivity, air pollution and road trauma (2). The transport and land planning sectors are part of the broader "built environment" (BE) concept, defined by the World Health Organization as 'Elements of the physical environment that are man-made, in contrast to the natural environment. The BE includes everything from metropolitan land-use patterns to urban transportation systems to individual buildings and the spaces around them' ( $3 \mathrm{p} 28)$. There is a growing body of evidence on the influence of the BE on health, specifically by either facilitating or hindering physical activity (PA) $(4,5)$. However BE initiatives that improve population levels of PA may expose individuals to increased risk from road trauma and air pollution (e.g. active travel programs). There is evidence to suggest that PA benefits outweigh these other health harms $(6,7)$.

For public initiatives in the transport and land use sectors, cost-benefit analysis (CBA) is the recommended method for the ex-ante appraisal of public policies in Australia and elsewhere $(8,9)$. As per Prest and Turvey, CBAs aim to 'maximise the present value of all benefits less that of all costs, subject to specified constraints' (10 p686 ). CBAs of public sector initiatives are commonly referred to as Social CostBenefit Analysis (11-13). The philosophical underpinnings of CBA are in welfare economics (14). Welfare economics is a branch of economics that aims to maximise societal welfare, interpreted as the sum of individuals' welfare (or utility) (15). CBA aims to support the decision-making process by providing relevant information, and is used together with other information to make decisions (13).

There are increasing calls for PA-related health benefits to be included in the appraisal of BE initiatives $(8,12,16)$. Lack of full consideration of health outcomes may misdirect allocation of resources towards developments that do not facilitate PA (e.g. motorways, disconnected streets, isolated housing developments, etc.), which may lead to societally sub-optimal outcomes. The United States, Australia, New Zealand and countries in Europe already produce guidance for the incorporation of PA-related health benefits in CBAs of initiatives within the transport sector (17). For 
Australia, a range of values have been suggested for incorporation of PA-related health benefits in CBAs of transport initiatives. Such values are expressed on per kilometre basis and range from less than $A \$ 0.02$ to $A \$ 1.46(2016)(18,19)$ per kilometre cycled (Figure S1 supplementary material). For walking, values ranged from $\mathrm{A} \$ 1.79$ to $\mathrm{A} \$ 2.92(2016)(9,18)$ (Figure S2 supplementary material). The range of values identified can be explained by both differences in the benefits included in the evaluation (e.g. mortality and morbidity, healthcare costs and productivity gains) and differences in the methods used to quantify those benefits. In Table S1 of the supplementary material we present a summary of the methods used to estimate per kilometre monetised values. These values are easily applicable to transport interventions with an active travel component where estimates of additional kilometres walked or cycled are available or can be made (see Table S2 in the supplementary material). However, these estimates were produced in the grey literature, and have not been scrutinized in a formal peer-review process. In addition, per kilometre estimates from the literature are applicable specifically in the transport sector, rather than the BE more broadly. It would therefore be useful to link the health benefits directly to characteristics of the BE (such as density, design characteristics and diversity of land use), where estimates of the effects are not readily translated into increases in kilometres travelled.

The aim of this study was to explore a method and a range of values that could incorporate monetised PA-related health benefits in CBAs assessing a broad range of $B E$ initiatives. In addition, we used our methods to produce monetised values of the PA health gains per kilometre walked and cycled for comparison with existing estimates from the Australian 'grey literature'.

\section{Methods}

The work presented here is based on the framework depicted in Figure $1(4,20)$. First, we systematically reviewed Australian contemporary literature for measures of the association between BE attributes and PA outcomes (4). Secondly, we translated effect sizes (e.g. odd ratios, beta coefficients) reported in our systematic review into average minutes of PA per week per adult living in a neighbourhood where an attribute of the BE changes (20). Minutes of PA per week (walking and cycling) as 
well as times per week are the most commonly collected type of data for studies of PA within the neighbourhood area (21). We grouped effect sizes reported in the literature according to five of the six 'D's proposed by Ewing and Cervero (22) (density, diversity of land uses, destinations, distance to transit and design) plus aggregated neighbourhood measures (i.e. walkability index). Third, we predicted annual average health-adjusted life years (HALYs) and healthcare costs per adult residing in a neighbourhood where there is a change in a feature of the BE using a mathematical model (20). HALYs are population health measures that incorporate mortality and quality of life (23). Healthcare costs include both savings from a reduction in physical inactivity related diseases and increases in costs due to health needs in the prolonged life years attributable to improvements in PA. Lastly, we multiplied predicted HALYs by the value of statistical life year (VSLY) and added net healthcare costs to produce overall monetised values linked to changes in features of the BE.

Detailed information about the literature review and calculation of average minutes of PA per week per adult living in a neighbourhood with a change in a BE feature can be found in Zapata-Diomedi and Veerman (4), Zapata-Diomedi et al. (20). The mathematical model and monetisation of HALYs are discussed below. Lastly, for comparison with values published in the grey literature, we used our methods to estimate monetised values per kilometre walked and cycled.

As highlighted in the introduction, we aimed to explore a methodology, and our methods can be adapted to specific evaluation needs. We used estimates from the literature to derive the difference in minutes walking per week for alternative BE exposures. Using these estimates demonstrates how the proportional multi-state life table model can be used to produce monetised values that can be applied in CBAs of $B E$ interventions.

\section{Mathematical model}

Our mathematical model is based on the multi-cohort proportional multi-state life table Markov model (model) developed for the 'Assessing Cost-Effectiveness in 
Prevention' project (ACE Prevention) (24-27). The model consists of a life table, a separate section for each of the modelled diseases and a section for population impact fraction (PIF) calculations. We included five diseases related to low levels of PA (ischemic heart disease, ischemic stroke, type 2 diabetes, colon cancer and breast cancer in women (28-30)). We modelled 5-year age groups by sex in until everyone reaches the age of 100 or dies. The PIF 'relative risk shift' method was used to estimate the effect of changes in PA on disease incidence rates (31). Changes in incidence impact on prevalent numbers of cases in later years, and consequently on years lived with disability and mortality. HALYs represent life years adjusted for disability attributable to disease and injury. The 'relative risk shift' method for calculating the PIF requires estimates of PA prevalence at baseline and for the intervention scenario, and corresponding relative risks (RR) of physical inactivity-related diseases. The Australian Bureau of Statistics collects data on four types of PA undertaken in the previous week by age and sex: walking for transport, walking for recreation, moderate PA (excluding walking) and vigorous PA (32). From this we derived PA scores by multiplying mean time spent in each of the aforementioned PA categories by an assigned metabolic equivalent value ${ }^{i}(M E T)$ (33) and then summing the results. These scores were used to categorise people into those who were highly active ( $\geq 1,600 \mathrm{MET}$-minutes)/wk.), those who met the recommended level of activity (600 to $<1,600 \mathrm{MET}$-minutes/wk.), those who were insufficiently active $<600 \mathrm{MET}$-minutes/wk.) and those who were inactive (0 METminutes/wk.) (28). We used Excel slope and intercept functions to fit RRs reported by Danaei et al (2009) with RRs as the dependent variable and mean METminutes/wk. per PA category at baseline as the independent variable. We used the slope and intercept parameters to estimate RRs for the modelled scenarios with MET-minutes per PA category changing accordingly.

Healthcare costs in the source study were expressed in 2010 values (20); we indexed these to 2016 using the Health Price Index (34). Results are based on the results in Zapata-Diomedi et al. (20) for a non-linear dose-response function for PA with health outcomes with diminishing returns for greater levels of PA (Table S3 and Figure S3 in the supplementary material). Healthcare costs and monetised HALYs were discounted at an annual rate of $3 \%(35)$. The model was set up in Excel and an 
uncertainty analysis was conducted with the Ersatz add-in tool (36). Uncertainty parameters are described in Table 2 from Zapata-Diomedi et al. (20).

\section{Monetisation of HALYs}

As per Mason et al. (2009) two methods may be applied to elicit the willingness to pay to derive the monetary value of a HALY ${ }^{1}$. One approach is to elicit its monetary value directly using survey methods. Another approach is to derive it from the monetary value of a prevented fatality used in the assessment of road infrastructure, also referred to as the value of statistical life (VSL). It is important to note that the VSL is also derived using survey methods. In what follows we explain the approach taken in this study, and further information for methods used to monetise HALYs are provided in the supplementary material.

Using the VSL for a prevented fatality is a convenient method that is widely used to monetise PA-related health benefits for incorporation in CBAs of active transport interventions (37). The application of this method in this study assumes that individuals value a reduction in the risk of mortality and morbidity attributable to low levels of PA similarly to those from road lifesaving interventions. This approach has been taken because eliciting the monetary value that the society places on a HALY for each individual intervention is time and resource consuming (38). In the supplementary material we expand on the derivation of the monetised value of a HALY from the value of statistical life year (VSLY) (derived from the value of statistical life).

\section{The Australian context}

In this research we used the VSLY to translate HALYs into monetised values. There is not a single, universally accepted VSLY in use for Australia. At the federal level, the Office of Best Practice Regulation (OBPR) recommends using a VSLY of $\mathrm{A} \$ 182,000$ in 2014 dollars in the preparation of CBAs for interventions that aim to reduce physical harm (OBPR, 39) $)^{i i}$.The OBPR estimate was recommended by Abelson $(40,41)$ after reviewing literature from Australia, the United States and European countries (VSLY 2003 value A $\$ 108,000)$. The VSLY in Abelson is based

\footnotetext{
1 The study refers to quality-adjusted life years, which is a type of population health measure that includes quality and quantity of life. We explain further in the supplementary material.
} 
on European values that used wage-risk and contingent valuation methods $(40,41)$. The VSLY was derived from a VSL of A $\$ 2.5$ million (2003) assuming a remaining life expectancy of 40 years with a $3 \%$ discount rate applied $(40,41)$. A life expectancy of 40 years is based on the average life expectancy and average age of the population (41).

\section{Using the multi-state life table approach to estimate per kilometre values}

To compare with estimates produced in the literature, and so provide a degree of "comparative validation" of our method, we also used the MSLT model to estimate monetised values per kilometre walked and cycled. We produced annual values assuming that walking one kilometre takes 12 minutes (at a speed of $5 \mathrm{~km}$ per hour) and cycling one kilometre takes 3 minutes ( $20 \mathrm{~km}$ per hour). We used MET rates from the PA compendium for walking and cycling (3.5 and 6.8) (33). We projected the monetised value for walking and cycling 1 kilometre per week divided by 52 (weeks in a year).

\section{Results}

\section{Monetised PA-health related values linked to changes to the built environment}

The range of estimated values is depicted in Figure 2. The interpretation is in terms of the annual average monetised PA-related health benefit for a person living in a neighbourhood where $B E$ changes are made, which could serve as reference values in CBAs of BE interventions. The monetised values presented here are specific to the changes in exposures to attributes of the $B E$ in the source studies. For example, while some estimates may be directly applicable to CBAs of BE initiatives such as an improvement in one transport destination. Others are rather specific, for example, an increase in street lights in a neighbourhood from 315 to 780 .

Overall, the results suggest that the greatest gains would be accrued from increasing destinations, walkability and attributes of design within the neighbourhood area. Annual PA-related health benefits worth up to $A \$ 70$ per resident were estimated for improvements in neighbourhood destinations. Improving neighbourhood walkability was estimated to be worth up to $A \$ 30$ and improvements in sidewalks availability (measure of design) up to $A \$ 22$ per adult resident. 
For further details on results, including uncertainty ranges and the magnitude of change in BE attributes and in PA, see Table S4 of the supplementary material. In our results, the monetised value of a HALY determines the overall value of PA. The savings in treatment cost of PA-related diseases are much smaller, and are negated by unrelated healthcare costs in added years of life.

Comparisons of results presented in Figure 2 per BE category should be made with caution. The effect estimates from the literature are based on a wide range of methods to measure exposure and outcomes. For instance, tested outcomes in the source studies include walking for transport, any walking, cycling for transport and recreational walking (Table S4 in the supplementary material).

FIGURE 2

\section{Monetised PA-related health values per kilometre walked and cycled}

Our results indicate that the value of PA-related health benefits associated with walking is $A \$ 0.98$ (95\% Uncertainty Interval (UI) 0.73 to 1.24) per kilometre. For cycling the benefits are worth $A \$ 0.62$ (95\% UI 0.46 to 0.79 ) per kilometre.

These estimates are in the lower end of the range compared with those proposed in the 'grey literature'. In Table 1 we present the values and summarise the outcomes in each of the estimates from the literature and our estimates. We cannot conclude that our values are lower due to a narrower inclusion of outcomes. Instead, this must be attributed to differences in methods or data.

TABLE 1

\section{Discussion}

\section{Summary}

We described a method and proposed a range of values that could be used for the incorporation of monetised PA-related health benefits in CBAs of BE initiatives. Our values directly link monetised health outcomes to the D categories (density, diversity of land uses, destinations, distance to transit and design) of the BE, plus aggregated neighbourhood measures (i.e. walkability index). Our proposed values can be used 
as ball-park estimates to assess specific urban planning scenarios where the planner aims to include wider societal benefits, such as health effects. We offer a flexible approach applicable to the Australian setting, however, given the availability of required data, it can be adapted to any context.

In addition, we estimated monetised PA-related health benefits per kilometre walked or cycled. To the authors' knowledge such values have not previously been published in the peer-reviewed literature.

\section{Strengths and limitations}

The main strength of this study is the robust prediction modelling approach that is based on the established and well-validated methods of the MSLT. It can be implemented in spreadsheets, which makes it transparent. The MSLT has been widely used for the assessment of health risk factors including high body mass index, tobacco smoking and physical inactivity $(27,42)$. Our modelling framework is more complex and demanding in terms of data inputs than those used in in the literature presented in the supplementary material for monetised values per kilometre walked and cycled. However, for Australia, the required data were available from the national statistics office and burden of disease studies. In this paper we focused on HALYs, but the model can be customised to include other outcomes such as disease incidence and prevalence, health-adjusted life expectancy and life years.

A number of limitations should be highlighted. Most of the literature assessing the association BE-PA indicates the odds of doing PA for a given exposure rather than an indication of magnitude that can be used to estimate health outcomes (43). Thus, assumptions were needed to translate effect sizes reported in the literature into minutes of PA per week, implying that our results should be only used as ballpark estimates (see Zapata-Diomedi et al. (2016)). In addition, most of the evidence for the BE-PA association is from cross-sectional studies, which by themselves do not prove causality (5). Furthermore, we assumed that a change in the BE translates into a change in overall PA, but it could be that the additional activity partly or fully replaces PA previously performed for other purposes, which would imply our results are biased upwards. Quasi-experimental studies of real interventions that measure total PA at multiple time points with case and control groups would provide a better 
indication of the effect of an intervention on total PA. Cluster randomised control trials would provide the highest quality of evidence for the effect of a BE intervention. Such trials are, however, not practical in this field. A further advancement would be to have complete information for the participants as to their initial levels of PA (e.g. inactive, insufficiently active and sufficiently active) so that an assessment of who benefits from the intervention could be made, and hence more precise estimates produced. In this study we assumed that everyone changes PA, with those already achieving 1,600 MET-minutes per week or more at baseline not receiving health benefits. This is supported by a recent quasi-experiment investigating the effect of transport infrastructure on walking, which found that the interventions encouraged walking in both active and inactive participants at baseline (44). Our proposed method only includes health benefits from changes in five physical inactivity related diseases. However, low levels of PA have also been found to be associated with mental health $(45,46)$. Other similar studies have also included the health pathway from PA to body mass index and health outcomes (47). Health related to body weight and mental health can be monetised in the same way as we did in this study with PA, via changes in the duration or health-related quality of life. The exact approach we used is limited by the set of health conditions that is defined in the Global Burden of Disease studies, but those are designed to be exhaustive and include mental health conditions.

\section{Conclusions}

Traditionally, CBAs of BE initiatives do not include potential PA-related health benefits, resulting in suboptimal welfare outcomes for society. This study provides a range of estimates for the value of PA-related health outcomes that can be used as reference values in CBAs of BE interventions for the Australian setting. Incorporating PA-related health in the appraisal of BE interventions may lead to healthier urban designs.

\section{Authors contribution}

BZ-D and JLV conceived the research question, designed the methods, conducted the analysis, wrote the manuscript and co-edited the paper. LG, AS, BGC and JLV critically reviewed and co-edited the paper. 


\section{Acknowledgments}

BZD, JLV, LG, BGC and AS are part of the NHMRC CRE in Healthy, Liveable Communities (\#1061404). BGC is supported by an NHMRC Senior Principal Research Fellowship (\#1107672) and VicHealth. BZD and LG are supported by the National Health and Medical Research Council (NHMRC) Centre of Research Excellence in Healthy, Liveable Communities (\#1061404). 


\section{References}

1. Giles-Corti B, Vernez-Moudon A, Reis R, Turrell G, Dannenberg AL, Badland H, et al. City planning and population health: a global challenge. The Lancet. 2016;388(10062):10-6.

2. Sallis JF, Bull F, Burdett R, Frank LD, Griffiths $P$, Giles-Corti $B$, et al. Use of science to guide city planning policy and practice: how to achieve healthy and sustainable future cities. The Lancet. 2016;388(10062):2936-47.

3. World Health Organization. Interventions on diet and physical activity: what works: summary report [Internet]. 2009 [Available from:

http://www.who.int/dietphysicalactivity/summary-report-09.pdf.

4. Zapata-Diomedi B, Veerman JL. The association between built environment features and physical activity in the Australian context: a synthesis of the literature. BMC Public Health. 2016;16(1):1-10.

5. McCormack G, Shiell A. In search of causality: a systematic review of the relationship between the built environment and physical activity among adults. Int J Behav Nutr Phys Act. 2011;8(1):125-.

6. Mueller N, Rojas-Rueda D, Cole-Hunter T, de Nazelle A, Dons E, Gerike R, et al. Health impact assessment of active transportation: a systematic review. Prev Med. 2015;76:103-14.

7. Doorley R, Pakrashi V, Ghosh B. Quantifying the health impacts of active travel: assessment of methodologies. Transport Reviews. 2015;35(5):559-82.

8. van Wee B, Börjesson M. How to make CBA more suitable for evaluating cycling policies. Transp Pol. 2015;44:117-24.

9. Transport for New South Wales. Principles and guidelines for economic appraisal of transport investment and initiatives Sydney: Transport for NSW; 2013 [Available from: http://www.transport.nsw.gov.au/sites/default/files/b2b/publications/tfnsw-principles-andguidelines-for-economic-appraisal-of-transport-initiatives.pdf.

10. Prest AR, Turvey R. Cost-benefit analysis: a survey. The Economic Journal. 1965;75(300):683735.

11. Dobes L, Argyrous G, Leung J. Social cost-benefit analysis in Australia and New Zealand. The state of current practice and what needs to be done: ANU Press; 2016.

12. Mulley $C$, Tyson R, McCue P, Rissel C, Munro C. Valuing active travel: Including the health benefits of sustainable transport in transportation appraisal frameworks. Res Transp Bus Manag. 2013;7:27-34.

13. Campbell HF, Brown RPC. Benefit-cost analysis : financial and economic appraisal using spreadsheets / Harry F. Campbell and Richard P.C. Brown. Cambridge: Cambridge : Cambridge University Press; 2003.

14. Drummond MF, Sculpher MJ, Torrance GW, O'Brien BJ, Stoddart GL. Methods for the economic evaluation of health care programmes. 3rd ed. United States: Oxford University Press; 2005.

15. Lange O. The foundations of welfare economics. Econometrica. 1942;10(3/4):215-28.

16. Litman T. Evaluating active transport benefits and costs: Victoria Transport Policy Institute; 2017 [Available from: http://www.vtpi.org/nmt-tdm.pdf. 
17. Mackie P, Worsley T, Eliasson J. Transport appraisal revisited. Res Transp Econ. 2014;47:3-

18.

18. Australian Transport Assessment and Planning (ATAP). Parameter Values 2016 [Available from: https://atap.gov.au/parameter-values/index.aspx.

19. PriceWaterhouseCoopers (PWC). Evaluation of the costs and benefits to the community of financial investment in cycling programs and projects in New South Wales, Final Report. Sydney: Roads and Traffic Authority of NSW and Department of Environment and Climate Change; 2009.

20. Zapata-Diomedi B, Mantilla Herrera AM, Veerman JL. The effects of built environment attributes on physical activity-related health and health care costs outcomes in Australia. Health \& Place. 2016;42:19-29.

21. Giles-Corti B, Timperio A, Cutt H, Pikora TJ, Bull FC, Knuiman M, et al. Development of a reliable measure of walking within and outside the local neighborhood: RESIDE's Neighborhood Physical Activity Questionnaire. Prev Med. 2006;42.

22. Ewing R, Cervero R. Travel and the built environment: a meta-analysis. J Am Plann Assoc. 2010;76(3):265-94.

23. Gold MR, Stevenson D, Fryback DG. HALYs and QALYs and DALYs, oh my: similarities and differences in summary measures of population health. Annual review of public health. 2002;23(1):115-34.

24. Barendregt JJ, Van Oortmarssen GJ, Van Hout BA, Van Den Bosch JM. Coping with multiple morbidity in a life table. Math Popul Stud. 1998;7(1):29-49.

25. Barendregt JJ, Oortmarssen vGJ, Murray CJ, Vos T. A generic model for the assessment of disease epidemiology: the computational basis of DisMod II. Popul Health Metr. 2003;1(1):4-.

26. Cobiac $L$, Vos T, Barendregt JJ. Cost-effectiveness of interventions to promote physical activity: a modelling study. Plos Med. 2009;6(7):e1000110-e.

27. Vos T, Carter R, Barendregt JJ, C. M, Veerman J, Magnus A, et al. Assessing Cost-Effectiveness in Prevention (ACE-Prevention): Final Report. University of Queensland, Brisbane and Deakin University, Melbourne; 2010.

28. Danaei G, Ding EL, Mozaffarian D, Taylor B, Rehm J, Murray CJL, et al. The preventable causes of death in the United States: comparative risk assessment of dietary, lifestyle, and metabolic risk factors. PLoS Med. 2009;6(4):e1000058.

29. Bull FC, Armstrong TP, Dixon T, Ham S, Neiman A, Pratt M. Physical inactivity. Comparative quantification of health risks: Global and regional burden of disease due to selected major risk factors Volume 1. 2004:729 - 881.

30. Kyu HH, Bachman VF, Alexander LT, Mumford JE, Afshin A, Estep K, et al. Physical activity and risk of breast cancer, colon cancer, diabetes, ischemic heart disease, and ischemic stroke events: systematic review and dose-response meta-analysis for the Global Burden of Disease Study 2013. BMJ. 2016;354.

31. Barendregt JJ, Veerman JL. Categorical versus continuous risk factors and the calculation of potential impact fractions. J Epidemiol Community Health. 2010;64(3):209-12.

32. Australian Health Survey: Physical Activity, 2011-12 [Internet]. 2015 [cited 22 September 2015]. Available from:

http://www.abs.gov.au/ausstats/abs@.nsf/Lookup/D4495467B7F7EB01CA257BAC0015F593?opend ocument. 
33. Ainsworth BE, Haskell WL, Herrmann SD, Meckes N, Bassett DR, Jr., Tudor-Locke C, et al. 2011 compendium of physical activities: a second update of codes and MET values. Med Sci Sports Exerc. 2011;43(8):1575-81.

34. Australian Institute of Health and Welfare. Health expenditure Australia 2014-15. Canberra: AlHW; 2016.

35. Gold MR. Cost-effectiveness in health and medicine. New York: Oxford University Press; 1996.

36. Barendregt JJ. EpiGear International 2012 [Available from: http://www.epigear.com/index files/prevent.html.

37. Brown V, Zapata-Diomedi B, Moodie M, Veerman JL, Carter R. A systematic review of economic analyses of active transport interventions that include physical activity benefits. Transport Policy. 2016;45:190-208.

38. Mason H, Baker R, Donaldson D. Willingness to pay for a QALY: past, present and future. Expert review of pharmacoeconomics \& outcomes research. 2008;8(6):575-82.

39. Office of Best Practice Regulation (OBPR). Best Practice Regulation Guidance Note: Value of statistical life: Department of Prime Minister and Cabinet. Australian Goverment; 2014 [Available from:

https://www.dpmc.gov.au/sites/default/files/publications/Value of Statistical Life guidance note. pdf.

40. Abelson P. Establishing a monetary value for lives saved: issues and controversies Canberra: Office of Best Practice Regulation; 2008. Report No.: 2008-02.

41. Abelson P. The value of life and health for public policy. Economic Record. 2003;79(Special Issue):S2-S13.

42. Mytton O, Tainio M, Ogilvie D, Panter J, Cobiac LJ, Woodcock J. Impact of increases in physical activity on disease burden, considering the effect of increasing life expectancy and reduced incidence of disease: a multi-state lifetable modelling study. Eur J Epidemiol. 2017;32(3):235-50.

43. Boarnet MG, Greenwald M, McMillan TE. Walking, urban design, and health: toward a costbenefit analysis framework. J Plann Educ Res. 2008;27(3):341-58.

44. Panter J, Ogilvie D. Can environmental improvement change the population distribution of walking? J Epidemiol Community Health Forthcoming 2017. 2017.

45. Hamer M, Chida Y. Physical activity and risk of neurodegenerative disease: a systematic review of prospective evidence. Psychol Med. 2009;39(1):3-11.

46. Blondell SJ, Hammersley-Mather R, Veerman JL. Does physical activity prevent cognitive decline and dementia?: A systematic review and meta-analysis of longitudinal studies. BMC Public Health. 2014;14(1):510.

47. Brown V, Moodie M, Cobiac L, Herrera A, Carter R. Obesity-related health impacts of fuel excise taxation-an evidence review and cost-effectiveness study. BMC Public Health2017.

\footnotetext{
$i$ "The ratio of the work metabolic rate to the resting metabolic rate. One MET is defined as 1 $\mathrm{kcal} / \mathrm{kg} / \mathrm{hour}$ and is roughly equivalent to the energy cost of sitting quietly. A MET also is defined as oxygen uptake in $\mathrm{ml} / \mathrm{kg} / \mathrm{min}$ with one MET equal to the oxygen cost of sitting quietly, equivalent to 3.5 $\mathrm{ml} / \mathrm{kg} / \mathrm{min}$." 33. Ainsworth BE, Haskell WL, Herrmann SD, Meckes N, Bassett DR, Jr., Tudor-Locke
} 
C, et al. 2011 compendium of physical activities: a second update of codes and MET values. Med Sci Sports Exerc. 2011;43(8):1575-81.

ii The original document says: "Many regulations have the benefit of reducing the risk of injury, diseases or disability. One method to value these benefits is to adjust the value of statistical life year (which could be interpreted as the value of a year of life free of injury, disease and disability) by a factor that accounts for the type of injury, disease or disability" 39. Office of Best Practice Regulation (OBPR). Best Practice Regulation Guidance Note: Value of statistical life: Department of Prime Minister and Cabinet. Australian Goverment; 2014 [Available from:

https://www.dpmc.gov.au/sites/default/files/publications/Value_of_Statistical_Life_guidance_note.pdf. 


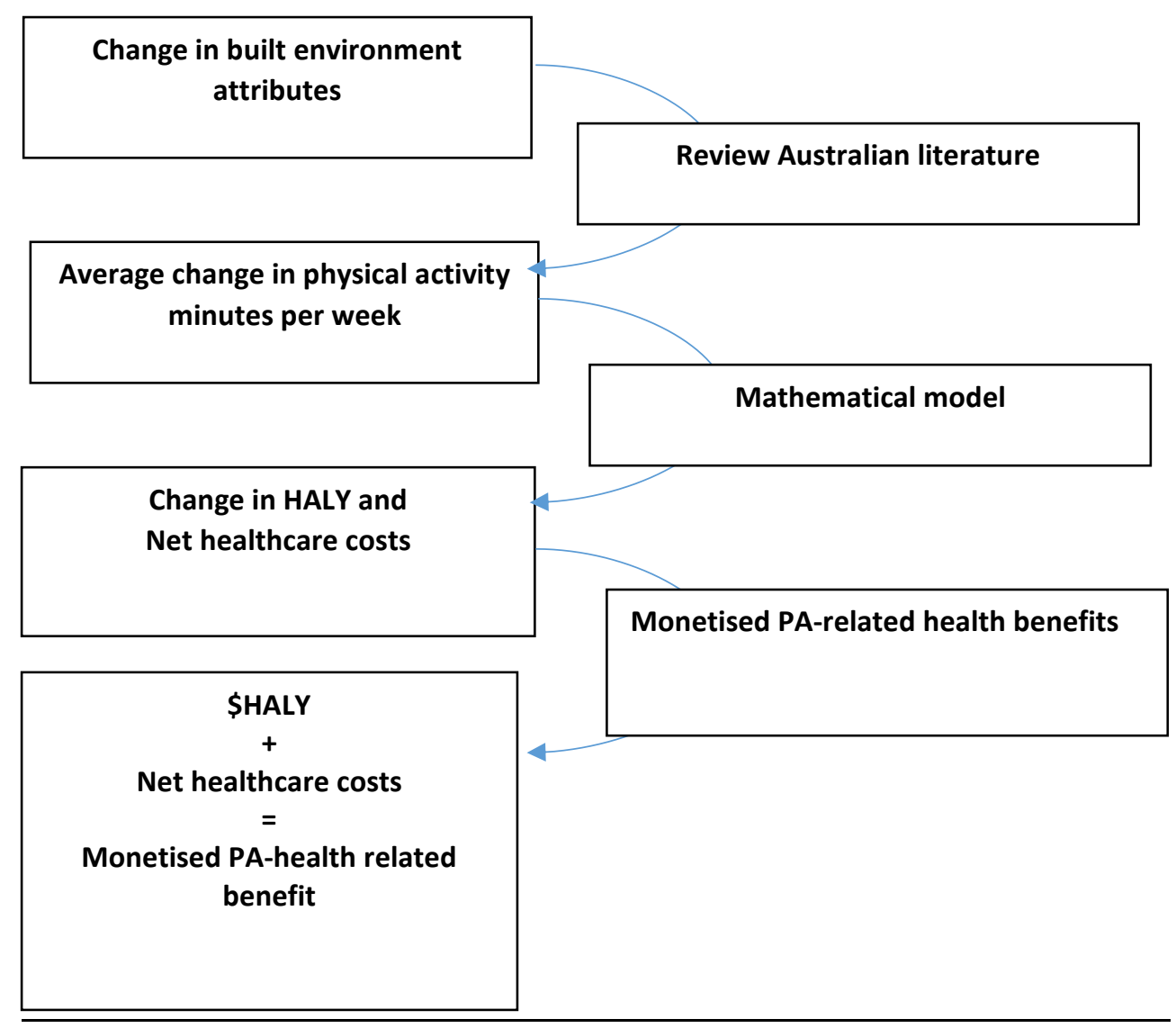

Figure 1 Study framework 


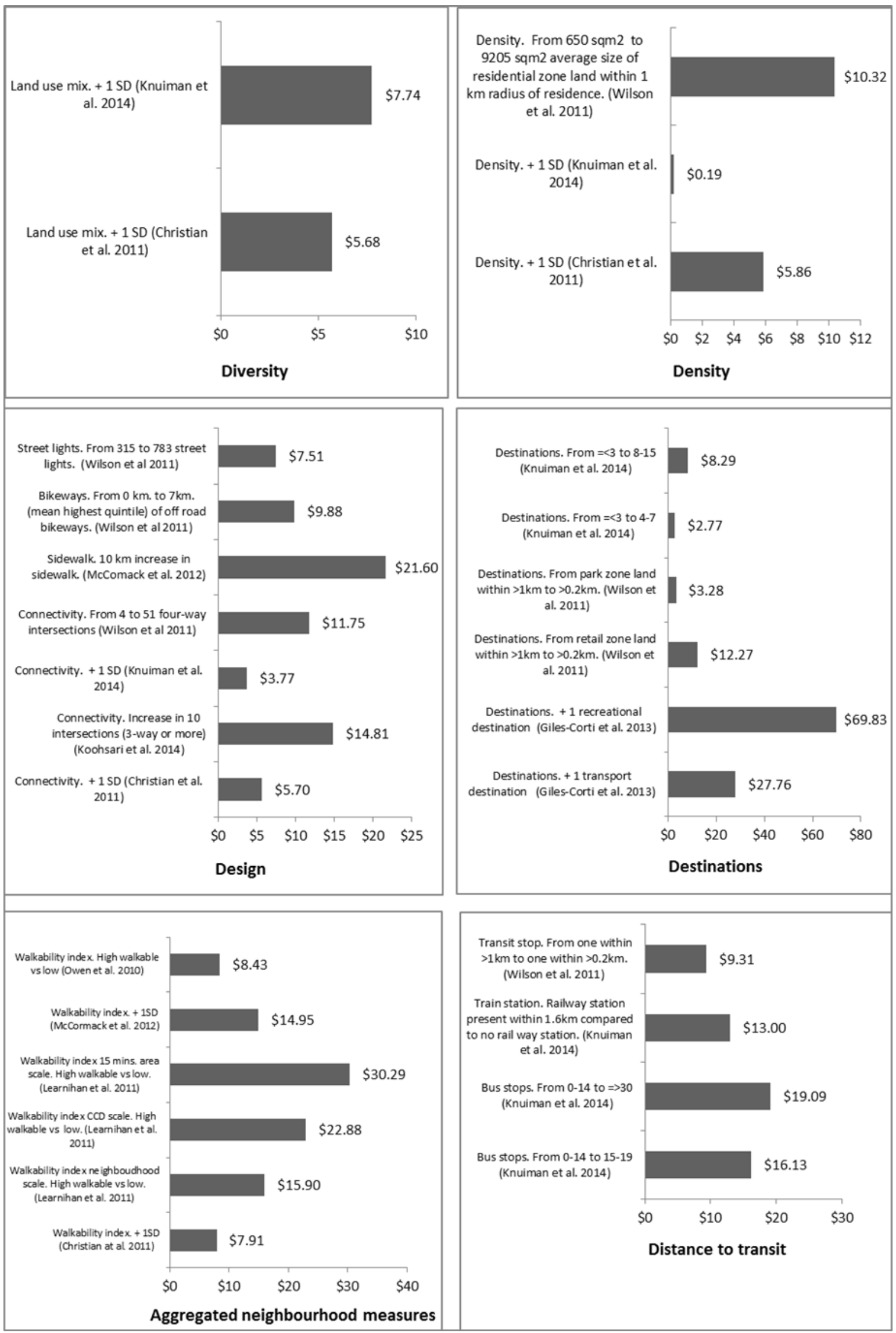

Figure 2 Monetised PA-related health benefits per year per adult living in a neighbourhood where built environment changes are made (A\$ 2016) ${ }^{a}$ ${ }^{a}$ Uncertainty intervals can be found in Table S3 of the supplementary material 